\title{
Sensitivity Analysis and Quantification of the Role of Governing Transport Mechanisms and Parameters in a Gas Flow Model for Low-Permeability Porous Media
}

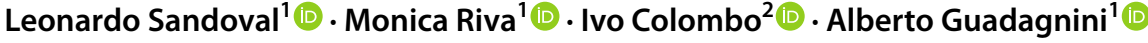

Accepted: 7 February 2022 / Published online: 3 March 2022

(c) The Author(s) 2022

\begin{abstract}
Recent models represent gas (methane) migration in low-permeability media as a weighted sum of various contributions, each associated with a given flow regime. These models typically embed numerous chemical/physical parameters that cannot be easily and unambiguously evaluated via experimental investigations. In this context, modern sensitivity analysis techniques enable us to diagnose the behavior of a given model through the quantification of the importance and role of model input uncertainties with respect to a target model output. Here, we rely on two global sensitivity analysis approaches and metrics (i.e., variance-based Sobol' indices and moment-based AMA indices) to assess the behavior of a recent interpretive model that conceptualizes gas migration as the sum of a surface diffusion mechanism and two weighted bulk flow components. We quantitatively investigate the impact of (i) each uncertain model parameter and (ii) the type of their associated probability distribution on the evaluation of methane flow. We then derive the structure of an effective diffusion coefficient embedding all complex mechanisms of the model considered and allowing quantification of the relative contribution of each flow mechanism to the overall gas flow.
\end{abstract}

\section{Article Highlights}

- Relative importance of parameters driving gas flow in low-permeability media is assessed.

- The influence of parameter probability distribution on gas flow statistics is appraised.

- A simple effective diffusion model embedding major methane flow mechanisms is derived.

Keywords Gas flow $\cdot$ Nanopores $\cdot$ Bulk flow $\cdot$ Methane $\cdot$ Sensitivity analysis

Leonardo Sandoval

rafaelleonardo.sandoval@polimi.it

1 Dipartimento di Ingegneria Civile e Ambientale, Politecnico di Milano, Piazza L. da Vinci 32, 20133 Milan, Italy

2 Geolog Technologies, Via Monte Nero 30, 20098 San Giuliano Milanese, Italy 


\section{List of Symbols}

$C_{\mathrm{g}} \quad$ Gas compressibility 1/MPa-MiniREFPROP

$C_{\mathrm{sc}} \quad$ Adsorbed concentration $\mathrm{kg} / \mathrm{m}^{3}$-Eq. 28

$d_{\mathrm{m}} \quad$ Gas molecule diameter $\mathrm{nm}-0.38$

$\partial p / \partial l \quad$ Gradient of gas pore pressure $\mathrm{MPa} / \mathrm{m}-0.1$

$D \quad$ Overall diffusion coefficient $\mathrm{m}^{2} / \mathrm{s}$-Eq. 15

$D_{\mathrm{s}} \quad$ Surface diffusion coefficient $\mathrm{m}^{2} / \mathrm{s}$-Eq. 25

$D_{\mathrm{k}} \quad$ Knudsen effective diffusion coefficient $\mathrm{m}^{2} / \mathrm{s}$-Eq. 16

$D_{\mathrm{ss}} \quad$ Surface effective diffusion coefficient $\mathrm{m}^{2} / \mathrm{s}$-Eq. 16

$D_{\mathrm{v}} \quad$ Slip flow effective diffusion coefficient $\mathrm{m}^{2} / \mathrm{s}$-Eq. 16

$J \quad$ Mass flux of gas per unit of area $\mathrm{T} /\left(\mathrm{m}^{2}\right.$ year $)$-Eq. 1

$J_{\mathrm{k}} \quad$ Knudsen diffusion $\mathrm{kg} /\left(\mathrm{m}^{2} \mathrm{~s}\right)$-Eq. 8

$J_{\mathrm{s}} \quad$ Surface diffusion $\mathrm{kg} /\left(\mathrm{m}^{2} \mathrm{~s}\right)$-Eq. 2

$J_{\mathrm{v}} \quad$ Slip flow $\mathrm{kg} /\left(\mathrm{m}^{2} \mathrm{~s}\right)$-Eq. 7

$K_{\mathrm{n}} \quad$ Knudsen number-Eq. 5

$M \quad$ Gas molar mass kg/mol- $1.6 \times 10^{-2}$

$p_{\mathrm{L}} \quad$ Langmuir pressure $\mathrm{MPa}-\mathrm{Eq} .22$

$p_{\mathrm{o}} \quad$ Atmospheric pressure $\mathrm{MPa}-0.1$

$r \quad$ Pore size nm-Eq. 19

$R \quad$ Universal gas constant $\mathrm{J} /(\mathrm{mol} \mathrm{K})-8,3144$

$r_{\text {ad }} \quad$ Thickness of adsorbed gas layer nm-Eq. 18

$w_{\mathrm{k}} \quad$ Knudsen diffusion flux weight factor-Eq. 4

$w_{\mathrm{v}} \quad$ Slip mass flux weight factor-Eq. 3

$Z \quad$ Gas deviation factor-MiniREFPROP

$\alpha \quad$ Rarefied effect coefficient for gas-Eq. 24

$\zeta_{\mathrm{ms}} \quad$ Correction factor of surface diffusion-Eq. 17

$\zeta_{\mathrm{mb}} \quad$ Correction factor bulk flow-Eq. 23

$\eta \quad$ Gas viscosity Pa s-MiniREFPROP

$\theta \quad$ Gas coverage of the geomaterial-Eq. 21

$\lambda \quad$ Mean free path of gas molecules m-Eq. 6

$\phi \quad$ Porosity-Eq. 20

\section{Introduction}

Methane is recognized as a potential energy source to assist transition to a carbon-free energy landscape (Hughes 2013), considerable reserves of methane being associated with subsurface reservoirs worldwide (U.S. Energy Information Administration 2015). After its generation, this gas typically accumulates in reservoir regions subdued to low-permeability layers (i.e., caprocks) that prevent its upward migration (Dembicki-Jr. 2017). Due to the partial sealing efficiency of caprocks, some amount of gaseous phase hydrocarbons might cross such barriers and reservoir gas can then be released into the overburden to (eventually) reach the surface (Schlömer and Krooss 1997; Schloemer and Krooss 2004). In this context, appropriate modeling approaches to quantify gas migration in low-permeability geomaterials can assist the appraisal of the feasibility of a methane recovery project.

A variety of models depicting gas movement in low-permeability geomaterials have been proposed (Wu et al. 2016; Sun et al. 2017; Rani et al. 2018; Wang et al. 2019). These models typically estimate the mass flow rate of gas as the result of a combination 
of various gas transport mechanisms taking place across the porous system. Parameters associated with these models, describing chemical, mechanical, flow, and transport features governing feedbacks between gas and the host rock matrix are always affected by uncertainty. The conceptual model of Wu et al. (2016) depicts the mass flow rate of a gas across a low-permeability medium as the sum of three key processes: (i) a surface diffusion, and two weighted bulk diffusion components corresponding to (ii) slip flow and (iii) Knudsen diffusion. This model takes into account changes in the porous system caused by mechanical deformation and adsorption/desorption dynamics. The model embeds numerous parameters which are typically estimated through (direct or indirect) laboratory-scale experiments. Considering the set of complex mechanisms involved, these types of experiments are costly, time demanding, and their results are prone to uncertainty. The latter is also related to the intrinsic difficulties linked to replicating operational field conditions at the laboratory scale as well as to the challenges stemming from transferability of results to heterogeneous field scale settings (Pan et al. 2010; Yuan et al. 2014; Tan et al. 2018).

Due to our still incomplete knowledge of the critical mechanisms driving gas movement in low-permeability media (Singh and Myong 2018; Javadpour et al. 2021) and the complexities associated with the estimation of model parameters, model outputs should be carefully analyzed considering all possible (aleatoric and epistemic) sources of uncertainty. In this sense, sensitivity analysis approaches are important tools enabling us to (i) quantify uncertainty, (ii) enhance our understanding of the relationships between model inputs and outputs, and (iii) tackle the challenges of model- and data-driven design of experiments (Dell'Oca et al. 2017). Hence, sensitivity analysis techniques may be effectively used in the context of methane flow modeling efforts to (i) quantify and rank the contribution of our lack of knowledge on each model parameter to the uncertainty associated with model outputs; (ii) identify model input-output relationships; and (iii) enhance the quality of parameter estimation workflows, upon focusing efforts on parameters with the highest influence to target model outputs (Saltelli et al. 2010; Dell'Oca et al. 2020). In cases where parameters associated with a model have already been estimated (e.g., through model calibration), the main purpose of a Global Sensitivity Analysis (GSA) is to assist quantification of the uncertainty still remaining after model calibration, thus guiding additional efforts for its characterization (e.g., Dell'Oca et al. (2020) and references therein). The probability density function (pdf) related to each model parameter at this step might differ from the one employed before model calibration and some model parameters might be associated with a reduced uncertainty. In cases where processes are described through black-box models, GSA can be employed to quantify the influence that the variability of hyperparameters embedded in these models can have on their outcomes. We note that if uncertainty of some model parameters is further constrained, for example through (stochastic) inverse modeling (e.g., Ceresa et al. (2021)), results of the uncertainty quantification might also change. In this work we illustrate the methodological framework and the workflow required for GSA of a methane flow model and provide the elements to perform such an analysis for diverse scenarios. In order to assist this process, we provide a repository with scripts developed during this work (see declaration section).

In this work, we rely on GSA approaches to study the behavior of the aforementioned gas migration model targeting low-permeability media. While previous works focus on only a few selected model parameters (Song et al. 2016; Wu et al. 2017; Sun et al. 2017), a comprehensive diagnosis of the system behavior based on rigorous and modern GSA approaches taking into account the way all model parameters influence model output uncertainty is still missing. Here, we do so by implementing two GSA techniques, respectively based on the evaluation of (i) the classical (variance-based) Sobol' indices (Saltelli 
and Sobol' 1995) and (ii) the recent moment-based GSA metrics proposed by Dell'Oca et al. (2017). We recall that GSA approaches relying on Sobol' indices are widely used to quantify the relative expected reduction of variance of the target model output due to the knowledge of (or conditioning on) a given parameter. These have been employed in several applications including diagnosis of models related to, e.g., flood risk assessment (Koks et al. 2015), overpressure risk assessment in sedimentary basins (Colombo et al. 2017), and energy storage (Xiao et al. 2021). A critical limitation of variance-based GSA methodologies is that the uncertainty of the output is considered to be completely characterized by its variance. Such an assumption can lead to an incomplete characterization of the system behavior. The moment-based GSA approach introduced by Dell'Oca et al. (2017) is designed to enhance our capability to evidence model behavior upon including the quantification of model parameter uncertainty on the (statistical) moments of the pdf of a model output of interest. As such, this comprehensive approach yields information enabling us to characterize various aspects of uncertainty, without being limited solely to the concept of variance. The ensuing indices (termed AMA indices, after the initials of the authors (Dell'Oca et al. 2017)) have been effectively employed in a variety of contexts, including geophysical analyses related to gravimetric responses due to pumping tests (Maina et al. 2021), biochemical degradation of compounds such as glyphosate in soils (la Cecilia et al. 2020), and groundwater flow, including its feedbacks with evapotranspiration (Bianchi Janetti et al. 2019; Maina and Siirila-Woodburn 2020).

This work is organized as follows: Sect. 2.1 briefly illustrates the complete model we consider to describe methane flow in low-permeability media. The main theoretical elements of the GSA approaches employed are described in Sect. 2.2. Key results of the GSA are presented in Sect. 3, where we also derive and discuss novel formulations describing an effective diffusive behavior and encapsulating all physical-chemical mechanism included in the full methane flow model described in Sect. 2.1. Finally, conclusions are drawn in Sect. 4.

\section{Materials and Methods}

\subsection{Gas Flow in Low-Permeability Media}

Models adopted to quantify gas migration in low-permeability media can be classified according to their complexity, in terms of, e.g., conceptualization and mathematical rendering of the embedded processes, as well as number of their characteristic parameters. Among existing models associated with a high degree of complexity and including multiple transport processes jointly contributing to the total gas migration across the system (Mehmani et al. 2013; Wu et al. 2015a, 2016, 2017; Sun et al. 2017; Zhang et al. 2018; Javadpour et al. 2021), here we consider the model of Wu et al. (2016). The selected model allows considering mechanical deformation as well as relevant features associated with real gases such as variations in the gas viscosity $(\eta)$, and the effects of the compressibility $\left(C_{\mathrm{g}}\right)$ and gas deviation $(Z)$ factors caused by pressure and temperature changes.

The model introduced by $\mathrm{Wu}$ et al. (2016) rests on a conceptual picture according to which the total mass flow rate of gas per unit of area $(J)$ is rendered through the sum of (i) a surface diffusion $\left(J_{\mathrm{S}}\right)$ and two weighted bulk diffusion components, corresponding to (ii) slip flow $\left(J_{\mathrm{v}}\right)$, and (iii) Knudsen diffusion $\left(J_{\mathrm{k}}\right)$, i.e., 


$$
J=J_{\mathrm{s}}+w_{\mathrm{v}} J_{\mathrm{v}}+w_{\mathrm{k}} J_{\mathrm{k}}
$$

The surface diffusion component is given by Wu et al. (2015b)

$$
J_{\mathrm{s}}=-\zeta_{\mathrm{ms}} \frac{D_{\mathrm{s}} C_{\mathrm{sc}}}{p} \frac{\partial p}{\partial l}
$$

where $p$ is (gas) pore pressure and $\frac{\partial p}{\partial l}$ represents the strength of the driving force through the system, corresponding to the spatial gradient of gas pore pressure. The (dimensionless) coefficient $\zeta_{\mathrm{ms}}$ is intended to take into account the possibility of applying the model (originally developed for capillary tubes) to a complex pore space and is defined in Eq. (17) of the Appendix where it is shown that $\zeta_{\mathrm{ms}}$ depends on porosity $(\phi)$, tortuosity $(\tau)$, pore size ( $r$ ) (i.e., pore radius), and gas coverage on the geomaterial $(\theta)$. The term $D_{\mathrm{s}}$ in Eq. (2) is the surface diffusion coefficient, which is expressed (as shown in Eq. (25)) in terms of gas temperature $(T)$, isosteric adsorption heat of the geomaterial $(\Delta H)$, a parameter $(\kappa)$ related to the blockage/migration ratio of the adsorbed molecules, and $\theta$. Finally, $C_{\mathrm{sc}}$, defined in Eq. (28), is the adsorbed concentration, which in turn depends on $\theta$ and on the gas molecule diameter $\left(d_{\mathrm{m}}\right)$.

The model proposed by $\mathrm{Wu}$ et al. (2016) allows representing the mechanical deformation of the pore space (in terms of variation of permeability and porosity with pressure) through power-law relationships and making use of the classical Kozeny-Carman equation. Here, we rest on their original model formulation, which naturally leads to Equations (19) and (20), clearly evidencing that both $r$ and $\phi$ evolve with $p$ as a function of a reference pore radius $\left(r_{\mathrm{o}}\right)$ and reference porosity $\left(\phi_{\mathrm{o}}\right)$, respectively.

The weight coefficients of the slip flow $\left(w_{\mathrm{v}}\right)$ and Knudsen diffusion $\left(w_{\mathrm{k}}\right)$ components in Eq. (1) are given by (Wu et al. 2016)

$$
\begin{gathered}
w_{\mathrm{v}}=\frac{1}{1+K_{\mathrm{n}}}, \\
w_{\mathrm{k}}=\frac{1}{1+1 / K_{\mathrm{n}}} .
\end{gathered}
$$

Here, $K_{\mathrm{n}}$ is the (dimensionless) Knudsen number defined as

$$
K_{\mathrm{n}}=\frac{\lambda}{2 r}
$$

with

$$
\lambda=\frac{\eta}{p} \sqrt{\frac{\pi Z R T}{2 M}},
$$

where $M$ and $R$ are the gas molar mass and universal constant, respectively. Note that $K_{\mathrm{n}}$ relates the mean free path of the gas molecules $(\lambda)$ to a representative length of the system (Civan 2010), here taken as the pore diameter.

The slip flow component is dominant in systems where $K_{\mathrm{n}}<0.1$ (Ziarani and Aguilera 2012) and can be evaluated as (Karniadakis et al. (2005); Wu et al. (2016)) 


$$
J_{\mathrm{v}}=-\zeta_{\mathrm{mb}} \frac{r^{2} p M}{8 \eta Z R T}\left(1+\alpha K_{\mathrm{n}}\right)\left(1+\frac{4 K_{\mathrm{n}}}{1+K_{\mathrm{n}}}\right) \frac{\partial p}{\partial l} .
$$

Here, $\zeta_{\mathrm{mb}}$ is intended to take into account the possibility of applying the slip flow formulation (7) to a complex pore space (see Eq. (23)) and $\alpha$ is the rarefied effect coefficient for a real gas which, according to Karniadakis et al. (2005), is evaluated through Eq. (24).

The Knudsen flow component is dominant in systems where $K_{\mathrm{n}}>10$ (Ziarani and Aguilera 2012) and is evaluated as (Darabi et al. (2012); Liu et al. (2016))

$$
J_{\mathrm{k}}=-\frac{2}{3} \zeta_{\mathrm{mb}} r \delta^{D_{\mathrm{f}}-2}\left(\frac{8 Z M}{\pi R T}\right)^{1 / 2} \frac{p}{Z} C_{\mathrm{g}} \frac{\partial p}{\partial l} .
$$

Here, $D_{\mathrm{f}}$ represents the fractal dimension of the pore surface and $\delta$ denotes the ratio between $d_{\mathrm{m}}$ and $r$.

We conclude by noting that the model here described includes a total of 15 parameters, which are related to the richness of physical processes embedded therein (See also Sect. 3.3). All quantities here introduced are listed in Table 1 and in the list of symbols and nomenclature Section.

\subsection{Global Sensitivity Analysis}

We perform a rigorous sensitivity analysis of the model illustrated in Sect. 2.1 to diagnose its behavior with reference to the estimate of methane flow as driven by imperfect knowledge of the associated parameters. Here, we note that uncertainties associated with the selection of the interpretative model is not analyzed. GSA can also be tailored to consider

\begin{tabular}{|c|c|c|c|}
\hline Parameter - (Units) - Symbol & Range (CV\%) & Criteria for the support & Reference \\
\hline Reference pore radius - $(\mathrm{nm})-r_{\mathrm{o}}$ & $2-100(55)$ & Literature & Wu et al. (2016) \\
\hline Reference porosity - (-) - $\phi_{\mathrm{o}}$ & $0.005-0.1(52)$ & Literature & Li et al. (2006) \\
\hline Pore pressure - $(\mathrm{MPa})-p$ & $0.5-50(57)$ & Literature & Wu et al. (2016) \\
\hline Tortuosity - (-) - $\tau$ & $2.8-5.8(20)$ & Literature & Mohd Amin et al. (2014) \\
\hline Temperature - $(\mathrm{K})-T$ & $337-473(10)$ & Literature & Chiquet et al. (2007) \\
\hline Overburden pressure $-(\mathrm{MPa})-p_{c}$ & $51-90(16)$ & Literature & Chiquet et al. (2007) \\
\hline Porosity exponent $-(-)-q$ & $0.014-0.056(35)$ & Literature & Dong et al. (2010) \\
\hline Pore radius exponent $-(-)-t$ & $0.02-0.04(19)$ & Literature & Dong et al. (2010) \\
\hline Block/migration ratio $-(-)-\kappa$ & $0.1-2(52)$ & Literature & Wu et al. (2015b) \\
\hline Fractal dimension - (-) - $D_{\mathrm{f}}$ & $2.1-2.9(9)$ & Theoretical limits & Coppens (1999) \\
\hline $\begin{array}{l}\text { Isosteric adsorption heat - }(\mathrm{J} / \\
\text { mol })-\Delta H\end{array}$ & $12,000-16,000(8)$ & Literature & Wu et al. (2015b) \\
\hline $\begin{array}{l}\text { Reference Langmuir pressure - } \\
\quad(\mathrm{Pa})-p_{L_{\mathrm{o}}}\end{array}$ & $41-128(30)$ & $\mathrm{CV}$ & Wu et al. (2015b) \\
\hline Parameter - (-) - $\alpha_{0}$ & $1.02-1.36(8)$ & Literature & Karniadakis et al. (2005) \\
\hline Parameter - (-) - $\alpha_{1}$ & $2-6(30)$ & $\mathrm{CV}$ & Karniadakis et al. (2005) \\
\hline Parameter - (-) - $\beta$ & $0.2-0.6(30)$ & $\mathrm{CV}$ & Karniadakis et al. (2005) \\
\hline
\end{tabular}

Table 1 Ranges of variability for the methane migration model uncertain parameters considered in the GSA

Values of the coefficient of variation, criteria for the selection of the range of variability, and references considered for the definition of each range of variability are also listed 
quantification of uncertainty of model outputs in the presence of uncertain interpretive models. In this context, uncertainty of a target variable which might result from the use of a collection of interpretive (conceptual and mathematical) models could be assessed upon relying, for example, on the approach illustrated by Dell'Oca et al. (2020). Our analysis is intended to yield a robust quantification of the relative importance of uncertain model parameters to a model output of interest. As mentioned in the Introduction, we rely on two GSA approaches, corresponding to (i) the classical variance-based technique grounded on the evaluation of the well-known Sobol' indices (Saltelli and Sobol' 1995) and (ii) the moment-based GSA framework introduced by Dell'Oca et al. (2017).

Model parameters are treated as statistically independent, as the amount of available information does not enable us to clearly identify cross-correlations among parameters and to quantify joint distributions. We consider three differing characterizations of pdf describing uncertainty of model parameters: $(a)$ all parameters are represented through uniform pdfs, $(b)$ all parameters are represented by truncated Gaussian pdfs, and (c) the reference pore radius is characterized by a (truncated) log-normal pdf, while all remaining parameters are associated with uniform pdfs. Case $a$ is representative of an approach where information on the considered parameters is limited so that all parameter values within the identified range of variability are equally weighted in the analysis (other studies relying on the same assumption include, e.g., Ciriello et al. (2013), Laloy et al. (2013), Sochala and Le Maître (2013), Bianchi Janetti et al. (2019), Dell'Oca et al. (2020)). Case $b$ is implemented as an alternative uninformed case, making use of the widely adopted hypothesis that model parameters are normally distributed. Case $c$ takes advantage of the findings of Naraghi et al. (2018) who provide some experimental evidence suggesting that the pdf of pore radii in shales can be interpreted through a log-normal model. Our choice of performing sensitivity analyses according to configurations associated with diverse pdfs characterizing uncertain model parameters enables us to analyze the influence of model parameter pdf (which is generally unknown a priori) on the results of the GSA and, ultimately, on gas flow forecasting.

Considering the computational cost associated with multiple model evaluations (corresponding to $10^{-4}$ seconds per simulation on an Intel Xeon Gold $6148 \mathrm{CPU} @ 2.4 \mathrm{GHz}$ ) required for these analyses and the corresponding cost for random sampling across the considered high dimensionality model parameter space, our analyses rest on $10^{8}$ model evaluations. The latter has been deemed to constitute an acceptable trade-off between the need to obtain stable results and computational efforts (details not shown). The pressure gradient acting on the system is set as a given boundary condition (and equal to $0.1 \mathrm{MPa} / \mathrm{m}$ ) in all test cases.

\subsubsection{Variance-Based Sobol' Indices}

Sobol' indices (Saltelli and Sobol' 1995) can assist the appraisal and quantification of the relative expected reduction of the variance of a target model output due to knowledge of (or conditioning on) a given model parameter, which would otherwise be subject to uncertainty. In this context, considering a model output $y$, which depends on $N$ random parameters collected in vector $\mathbf{x}=\left(x_{1}, x_{2}, \ldots, x_{N}\right)$ and defined within the space $\Gamma=\Gamma_{1} \times \Gamma_{2} \times \cdots \times \Gamma_{N}\left(\Gamma_{i}=\left[x_{i, \min }, x_{i, \max }\right]\right.$ corresponding to the support of the $i$ th parameter, $x_{i}$ ), the principal Sobol' index $S_{x_{i}}$ associated with a given model parameter $x_{i}$ is evaluated as 


$$
S_{x_{i}}=\frac{V\left[E\left[y \mid x_{i}\right]\right]}{V[y]} .
$$

Here, $E[\cdot]$ and $V[\cdot]$ represent expectation and variance operators, respectively; the notation $y \mid x_{i}$ denotes conditioning of $y$ on $x_{i}$. Note that $S_{x_{i}}$ describes the relative contribution to $V[y]$ due to variability of only $x_{i}$. Joint contributions of $x_{i}$ with other model parameters included in $\mathbf{x}$ to the variance of $y$ are embedded in the total Sobol' indices (details not shown). We recall that relying on Sobol' indices to diagnose the relative importance of uncertain model parameters to model outputs is tantamount to identifying uncertainty with the concept of variance of a pdf. As such, while Sobol' indices are characterized by a conceptual simplicity and straightforward implementation and use, they provide only limited information about the way variations of model parameters can influence the complete pdf of model outputs.

\subsubsection{Moment-Based AMA Indices}

The recent moment-based GSA approach proposed by Dell'Oca et al. (2017, 2020) rests on the idea that the quantification of the effects of model parameter uncertainty on various statistical moments of the ensuing pdf of model outputs can provide enhanced understanding of model functioning. Dell'Oca et al. (2017) introduce Moment-Based sensitivity metrics (termed AMA indices) according to which one can evaluate the influence of uncertain model parameter on key elements of the model output pdf, as embedded in its associated statistical moments. The AMA indices are defined as follows (Dell'Oca et al. (2017)):

$$
\operatorname{AMA} M_{x_{i}}=\frac{1}{|M[y]|} E\left[\left|M[y]-M\left[y \mid x_{i}\right]\right|\right] .
$$

Here, AMA $M_{x_{i}}$ represents the indices associated with a model parameter $x_{i}$ and a given statistical moment $M$ of the pdf of model output $y$ (considering the first four statistical moments of $y, M=E$ for the mean, $M=V$ for the variance, $M=\gamma$ for the skewness, and $M=k$ for the kurtosis). The AMA indices are intended to quantify the expected change of each statistical moment of $y$ due to our knowledge of $x_{i}$. Large values of these indices indicate that variations of the associated parameter strongly affect the statistical moments of $y$.

\section{Results and Discussion}

\subsection{GSA of Methane Flow Model}

The 15 uncertain model parameters of model (1) are considered to vary across the support defined through the ranges of variability listed in Table 1 . These ranges have been designed upon considering available literature references (values typically employed for the model parameters in low-permeability geomaterials). With reference to three of the model parameters i.e., $L_{p_{0}}, \alpha_{1}$, and $\beta$, only very limited information is available from the literature, to the best of our knowledge (Karniadakis et al. 2005). Thus, we take the values considered by $\mathrm{Wu}$ et al. (2016) and Karniadakis et al. (2005) as the centers of corresponding ranges of variability. We then consider their (uniform) distributions to be characterized by a given 
coefficient of variation (that we set as 30\%), thus enabling us to imprint these parameters with a sufficiently broad range of variability, which is also consistent with the degree of variability documented for the remaining uncertain parameters (see Table 1). Finally, we allow the fractal dimension $D_{\mathrm{f}}$ to vary within its theoretical bounds (i.e., $2<D_{\mathrm{f}}<3$ ) (Coppens 1999; Coppens and Dammers 2006). Methane properties (such as viscosity, compressibility, and deviation factor) are estimated using miniREFPROP (Lemmon et al. 2018), a tool that incorporates equations of state for a variety of gas species. With reference to methane miniREFPROP relies on the equation of state proposed by Setzmann and Wagner (1991).

Table 2 lists the moment-based GSA indices related to mean (AMA $E_{x_{i}}$ ), variance $\left(\mathrm{AMA} V_{x_{i}}\right)$, skewness $\left(\mathrm{AMA} \gamma_{x_{i}}\right)$, and kurtosis $\left(\mathrm{AMA} k_{x_{i}}\right)$ of $J$ as well as the principal Sobol' indices $\left(S_{x_{i}}\right)$ evaluated for methane flow rate values rendered by Eq. (1) for the case in which all model parameters are modeled as independent and identically distributed random variables, each characterized by a uniform pdf (Case $a$ ).

While the strength of the influence of the reference pore radius $\left(r_{\mathrm{o}}\right)$ on the model output is not the same for the (first four) statistical moments, the AMA indices clearly suggest that conditioning on $r_{\mathrm{o}}$ has (overall) the strongest impact on the first four statistical moments of methane flow. This is then followed by reference porosity, pore pressure, tortuosity, and temperature. While the remaining model uncertain parameters still exert some influence on the (first four) statistical moments of $J$ (as evidenced by the non-zero values of AMA indices), the strength of their influence can be considered as marginal when compared to the above mentioned quantities, which are seen to be key in driving the main features of the pdf of methane flow. In the following, we denote as most influential parameters for metrics AMA $M_{x_{i}}$ or $S_{x_{i}}$ all parameters $x_{i}$ corresponding to AMA $M_{x_{i}} / \sum_{x_{i}} \mathrm{AMA} M_{x_{i}} \geq 5 \%$ or $S_{x_{i}} / \sum_{x_{i}} S_{x_{i}} \geq 5 \%$, respectively. Parameters identified as most influential by each of these metrics are reported in bold in Table 2. Values of the Sobol' principal indices are generally

Table 2 Moment-based GSA indices AMA $M_{x_{i}}$ and Sobol' principal indices $S_{x_{i}}$ for all $x_{i}$ parameters included in Eq. (1)

\begin{tabular}{llllll}
\hline$x_{i}$ & $\mathrm{AMA} E_{x_{i}}$ & $\mathrm{AMA} V_{x_{i}}$ & $S_{x_{i}}$ & $\mathrm{AMA} \gamma_{x_{i}}$ & $\mathrm{AMA} k_{x_{i}}$ \\
\hline$r_{\mathrm{o}}$ & $\mathbf{0 . 7 2 8}$ & $\mathbf{0 . 7 9 8}$ & $\mathbf{0 . 4 1 7}$ & $\mathbf{0 . 5 6 2}$ & $\mathbf{0 . 7 5 7}$ \\
$\phi_{\mathrm{o}}$ & $\mathbf{0 . 4 5 3}$ & $\mathbf{0 . 6 4 3}$ & $\mathbf{0 . 1 6 0}$ & $\mathbf{0 . 3 4 5}$ & $\mathbf{0 . 4 6 4}$ \\
$p$ & $\mathbf{0 . 3 3 5}$ & $\mathbf{0 . 4 8 4}$ & $\mathbf{0 . 0 9 1}$ & $\mathbf{0 . 2 0 8}$ & $\mathbf{0 . 4 7 6}$ \\
$\tau$ & $\mathbf{0 . 1 8 1}$ & $\mathbf{0 . 3 5 6}$ & 0.026 & $\mathbf{0 . 1 1 4}$ & $\mathbf{0 . 2 1 3}$ \\
$T$ & 0.094 & $\mathbf{0 . 1 6 3}$ & 0.007 & 0.027 & 0.046 \\
$q$ & 0.061 & 0.119 & 0.003 & 0.011 & 0.022 \\
$t$ & 0.057 & 0.114 & 0.003 & 0.01 & 0.021 \\
$p_{c}$ & 0.028 & 0.063 & 0.001 & 0.008 & 0.014 \\
$\kappa$ & 0.010 & 0.005 & 0 & 0.004 & 0.007 \\
$\Delta H$ & 0.001 & 0.002 & 0 & 0.002 & 0.005 \\
$D_{\mathrm{f}}$ & 0.002 & 0.003 & 0 & 0.002 & 0.004 \\
$p_{L_{\mathrm{o}}}$ & 0.002 & 0.003 & 0 & 0.002 & 0.004 \\
$\alpha_{0}$ & 0.001 & 0.002 & 0 & 0.002 & 0.004 \\
$\alpha_{1}$ & 0.001 & 0.002 & 0 & 0.002 & 0.004 \\
$\beta$ & 0.001 & 0.002 & 0 & 0.002 & 0.004 \\
\hline
\end{tabular}

All model parameters are described by uniform pdfs (Case $a$ ). Values of each metric identifying the most influential parameters are reported in bold 

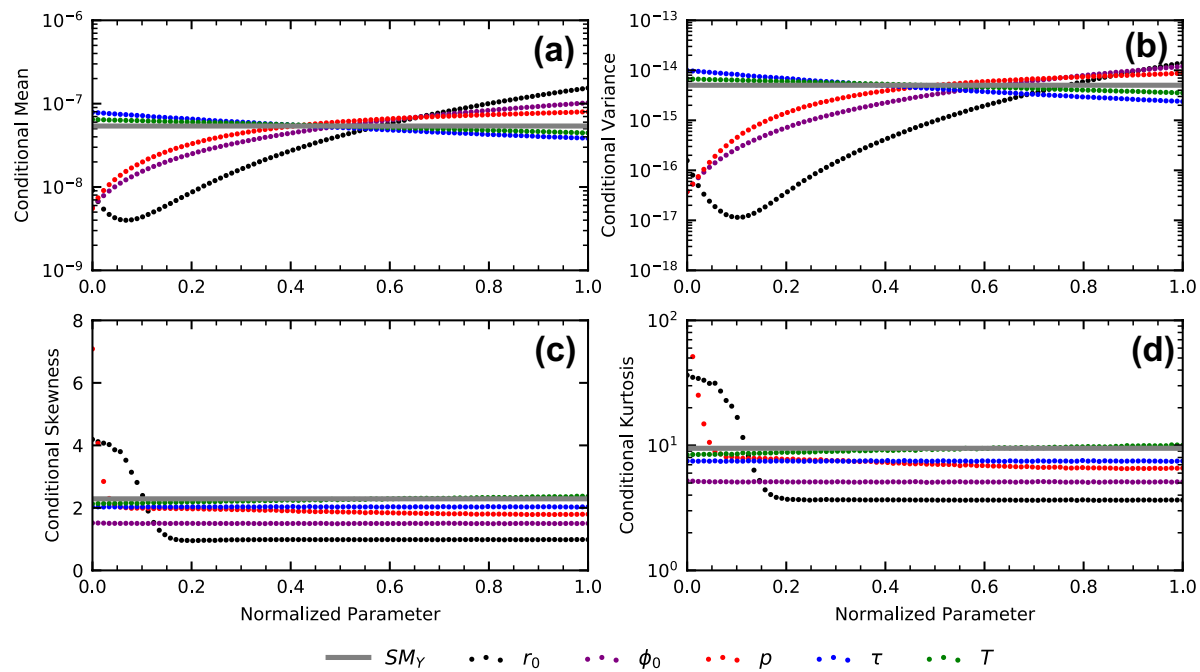

Fig. 1 First four statistical moments of methane flow $J\left(\mathrm{~T} / \mathrm{m}^{2}\right.$ year) conditional on values of the most influential model parameters (see Table 2): a expected value, b variance, c skewness, and d kurtosis. The corresponding unconditional moments (i.e., $\mathrm{SM}_{Y}$ ) are also depicted (gray bold horizontal lines). Intervals of variation of the uncertain model parameters are rescaled within the unit interval for graphical representation purposes. All model parameters are described by uniform pdfs (Case $a$ )

consistent with the results stemming from the moment-based GSA, even as $\tau$ and $T$ are not identified as influential to the model output according to the Sobol' principal index. This result is consistent with the observation that conditional variance can be larger or smaller than its unconditional counterpart (see also Fig. 1b) in a way that its integral over $\Gamma_{T}$ vanishes. A similar effect associated with the principal Sobol' indices was identified by Dell'Oca et al. (2017) with reference to the Ishigami function, which is a widely used analytical benchmark in sensitivity studies.

Figure 1 depicts the first four statistical moments of $J$ conditioned on values of the five most influential uncertain parameters selected on the basis of Table 2. Uncertain parameters are normalized to span the unit interval, for ease of interpretation. Unconditional moments are also depicted as a reference. We note that, when considering conditioning on the model parameters which have been identified as non-influential according to the metrics employed, the difference between conditional and unconditional moments is negligible (details not shown).

As expected, conditioning on values of the reference pore radius $\left(r_{\mathrm{o}}\right)$ yields the most marked effects to all of the statistical moments considered (see black dotted curves in Fig. 1). Mean and variance of methane flow generally increase with $r_{\mathrm{o}}$. A minimum mean methane flow value is attained for $2<r_{\mathrm{o}}<15 \mathrm{~nm}$ (corresponding to the range of normalized values comprised between 0 and 0.15 in Fig. 1). The dominant transport mechanism for $r_{\mathrm{o}}<15 \mathrm{~nm}$ is surface diffusion, the strength of its contribution decreasing with increasing $r_{\mathrm{o}}$. As $r_{\mathrm{o}}$ increases, the strength of the contribution related to surface diffusion decreases faster than the corresponding increase of the slip flow contribution, thus resulting in a minimum value for the expected methane flow for values of the reference pore radius comprised in the aforementioned range. Otherwise, skewness and kurtosis (i) are affected by variations of the reference pore radius when the latter is smaller than $20 \mathrm{~nm}$ (corresponding to a normalized value of 0.18 ); and (ii) are generally constant for $r_{\mathrm{o}}>20$ 
nm. Nevertheless, we note that these (statistical) moments are still remarkably different from their unconditional counterparts even for large $r_{\mathrm{o}}$ values, thus evidencing the impact of acquired knowledge on $r_{\mathrm{o}}$ on reducing the asymmetry (as rendered by the skewness) and the peakedness and tailedness (i.e., the probability associated with extreme values, as rendered by the kurtosis) of the methane flow pdf.

Conditioning on pore pressure imprints variations to the statistics of the model output which are qualitatively similar to those associated with $r_{\mathrm{o}}$. Larger values of mean and variance of $J$ are linked to larger values of $p$. This result descends from the linear relationship between pore pressure and slip flow (Eq. (7)), the latter being the dominant mechanism in systems formed by larger pores. Conditional skewness and kurtosis are constant (albeit different from their unconditional counterpart) across most of the variability range of $p$, sharp variations of these quantities being associated with conditioning on low values of $p$ (i.e., corresponding to pore pressure values smaller than $10 \mathrm{MPa}$ ). Our findings about the influence of $p$ on $J$ are consistent with the results of Sun et al. (2017). These authors find that increasing values of pore pressure lead to an increase of apparent permeability (which is in turn linearly proportional to gas flow) for $r_{\mathrm{o}}>10 \mathrm{~nm}$. Wu et al. (2016) document a similar behavior due to the dominance of the slip flow component (which is proportional to $p$; see Eq. (7)) in systems characterized by large pores.

While the impact of reference porosity and tortuosity is not analyzed in any of the available previous studies (Wu et al. 2015b, 2016, 2017; Sun et al. 2017; Zhang et al. 2018), our results rank $\phi_{\mathrm{o}}$ and $\tau$ as the second and fourth most influential parameters in the evaluation of the pdf of $J$, respectively (see Table 2). The correction factors for bulk (Eq. (23)) and surface (Eq. (17)) diffusion flow increase linearly with reference porosity. Thus, increased values of $\phi_{\mathrm{o}}$ yield corresponding increases of the methane flow (and hence of its first two statistical moments) independent of the dominant transport mechanism. Conditional mean and variance of $J$ decrease with increasing values of tortuosity. This is in line with the observation that all gas transport mechanisms are characterized by an inverse proportionality between $J$ and $\tau$ through the correction factor which is related to these processes taking place within a porous domain. These elements are consistent with a physical picture according to which fluid flow rates across a porous geomaterial are expected to increase and decrease with increasing porosity and tortuosity, respectively. Unlike pore pressure and reference pore radius, conditioning on reference porosity and tortuosity yields a reduction of skewness and kurtosis of the pdf of $J$, whose conditional values remain constant independent of the value of $\phi_{\mathrm{o}}$ and/or $\tau$.

Conditioning on temperature $(T)$ affects the mean and variance of the methane flow pdf in a way which is qualitatively similar to the effect of tortuosity (albeit quantitatively to a lesser extent) due to the inverse proportionality between $J$ and $T$. Otherwise, the overall shape of the pdf of $J$ is not significantly influenced by the knowledge of $T$, values of conditional skewness and kurtosis practically coincide with their unconditional counterparts.

The results listed in Table 2 suggest that statistical moments of methane flow are virtually insensitive to the remaining parameters (i.e., 10 of the 15 model parameters). Therefore, setting any of these parameters at given values within the variability space considered in our analysis yields only minor changes in the prediction of $J$. In this context, our results suggest that methane flow can be assessed with an acceptable degree of reliability even in the presence of scarce information about several parameters embedded in Eq. (1) such as, e.g., the overburden pressure (i.e., $p_{c}$ ), the power-law exponents related to porosity (i.e., $q$ ) and pore radius (i.e., $t$ ), the fractal dimension of the pore surface (i.e., $D_{\mathrm{f}}$ ), or the isosteric adsorption heat of the geomaterial (i.e., $\Delta H$ ). Further to this, our results suggest the 
opportunity to prioritize allocation of resources to robust characterization of (in descending order) reference pore radius, reference porosity, pore pressure, tortuosity, and temperature.

\subsection{Impact of the Model Parameter pdfs on GSA Results}

In this section, we analyze the impact of the choice of model parameter distribution on the pdf of $J$. As described in Sect. 2.2, we compare the GSA outcomes obtained with a uniform pdf for all model parameters (Case $a$ ) and illustrated in Sect. 3.1 against those computed when (i) all model parameters are characterized through (truncated) Gaussian pdfs (Case $b$ ) and (ii) $r_{\mathrm{o}}$ is described by a (truncated) log-normal pdf while the remaining parameters are described as in Case $a$ (Case $c$ ). To provide a consistent comparison, Gaussian and lognormal pdfs are defined to honor the same mean and variance of the scenario associated with Case $a$.

Table 3 lists values of AMA and principal Sobol' indices for each of the parameters embedded in Eq. (1) for Case $b$. Results of Tables 3 and 2 are qualitatively similar, i.e., the GSA yields similar results considering a uniform or a (truncated) Gaussian pdf for all model parameters. Our results imbue us with confidence about the documented ranking of parameter importance, with reference pore radius, reference porosity, pore pressure, tortuosity, and temperature identified as the model parameters being the key drivers to the evaluation of the major features of the pdf of methane flow. Values of statistical moments of $J$ conditioned on model parameters for Case $b$ are very similar to those depicted in Fig. 1 for Case $a$ (details not shown).

Table 4 lists the AMA and the principal Sobol' indices associated with $J$ for Case $c$. In this case, it is even more evident that the uncertainty of $r_{\mathrm{o}}$ is strongly dominant on the evaluation of the pdf of methane flow. Additionally, the blockage/migration ratio of the

Table 3 Moment-based GSA indices AMA $M_{x_{i}}$ and Sobol' principal indices $S_{x_{i}}$ for all $x_{i}$ parameters included in Eq. (1)

\begin{tabular}{llllll}
\hline$x_{i}$ & $\mathrm{AMA} E_{x_{i}}$ & $\mathrm{AMA} V_{x_{i}}$ & $S_{x_{i}}$ & $\mathrm{AMA} \gamma_{x_{i}}$ & $\mathrm{AMA} k_{x_{i}}$ \\
\hline$r_{\mathrm{o}}$ & $\mathbf{0 . 7 8 7}$ & $\mathbf{0 . 8 2 8}$ & $\mathbf{0 . 7 6 1}$ & $\mathbf{0 . 6 0 8}$ & $\mathbf{0 . 6 9 2}$ \\
$\phi_{\mathrm{o}}$ & $\mathbf{0 . 4 5 2}$ & $\mathbf{0 . 6 7 4}$ & $\mathbf{0 . 2 4 2}$ & $\mathbf{0 . 3 0 6}$ & $\mathbf{0 . 4 0 2}$ \\
$p$ & $\mathbf{0 . 3 2 1}$ & $\mathbf{0 . 4 8 1}$ & $\mathbf{0 . 1 3 1}$ & $\mathbf{0 . 1 5 2}$ & $\mathbf{0 . 3 0 2}$ \\
$\tau$ & $\mathbf{0 . 1 8 2}$ & $\mathbf{0 . 3 6 3}$ & 0.041 & $\mathbf{0 . 0 8 8}$ & $\mathbf{0 . 1 5 7}$ \\
$T$ & $\mathbf{0 . 1 0 0}$ & $\mathbf{0 . 1 7 8}$ & 0.012 & 0.027 & 0.042 \\
$q$ & 0.063 & 0.122 & 0.005 & 0.010 & 0.018 \\
$t$ & 0.059 & 0.117 & 0.004 & 0.009 & 0.016 \\
$p_{c}$ & 0.025 & 0.056 & 0.001 & 0.006 & 0.011 \\
$\kappa$ & 0.007 & 0.005 & 0 & 0.005 & 0.008 \\
$\Delta H$ & 0.001 & 0.002 & 0 & 0.003 & 0.007 \\
$D_{\mathrm{f}}$ & 0.001 & 0.003 & 0 & 0.002 & 0.005 \\
$p_{L_{\mathrm{o}}}$ & 0.001 & 0.002 & 0 & 0.003 & 0.006 \\
$\alpha_{0}$ & 0.001 & 0.002 & 0 & 0.002 & 0.005 \\
$\alpha_{1}$ & 0.001 & 0.002 & 0 & 0.003 & 0.006 \\
$\beta$ & 0.001 & 0.002 & 0 & 0.003 & 0.006 \\
\hline
\end{tabular}

All model parameters are described by truncated Gaussian distributions (Case $b$ ). Values of each metric identifying the most influential parameters are reported in bold 
Table 4 Moment-based GSA indices AMA $M_{x_{i}}$ and Sobol' principal indices $S_{x_{i}}$ for all $x_{i}$ parameters included in Eq. (1)

\begin{tabular}{llllll}
\hline$x_{i}$ & $\mathrm{AMA} E_{x_{i}}$ & $\mathrm{AMA} V_{x_{i}}$ & $S_{x_{i}}$ & $\mathrm{AMA} \gamma_{x_{i}}$ & $\mathrm{AMA} k_{x_{i}}$ \\
\hline$r_{\mathrm{o}}$ & $\mathbf{3 . 3 3 2}$ & $\mathbf{3 . 6 4 9}$ & $\mathbf{2 . 8 0 3}$ & $\mathbf{0 . 7 8 8}$ & $\mathbf{0 . 8 8 3}$ \\
$\phi_{\mathrm{o}}$ & $\mathbf{0 . 4 5 2}$ & $\mathbf{0 . 6 9 0}$ & 0.064 & $\mathbf{0 . 2 1 2}$ & $\mathbf{0 . 4 0 4}$ \\
$p$ & 0.192 & $\mathbf{0 . 5 0 7}$ & 0.012 & $\mathbf{0 . 1 5 2}$ & $\mathbf{0 . 2 6 3}$ \\
$\tau$ & 0.181 & $\mathbf{0 . 3 5 8}$ & 0.011 & $\mathbf{0 . 0 7 0}$ & $\mathbf{0 . 1 6 7}$ \\
$T$ & 0.090 & 0.173 & 0.003 & 0.024 & 0.050 \\
$q$ & 0.063 & 0.121 & 0.001 & 0.008 & 0.020 \\
$t$ & 0.041 & 0.112 & 0.001 & 0.008 & 0.020 \\
$p_{c}$ & 0.023 & 0.061 & 0 & 0.007 & 0.016 \\
$\kappa$ & 0.112 & 0.010 & 0.005 & 0.021 & 0.027 \\
$\Delta H$ & 0.002 & 0.006 & 0 & 0.004 & 0.010 \\
$D_{\mathrm{f}}$ & 0.02 & 0.008 & 0 & 0.011 & 0.018 \\
$p_{L_{\mathrm{o}}}$ & 0.025 & 0.007 & 0 & 0.011 & 0.017 \\
$\alpha_{0}$ & 0.002 & 0.006 & 0 & 0.005 & 0.013 \\
$\alpha_{1}$ & 0.002 & 0.006 & 0 & 0.005 & 0.012 \\
$\beta$ & 0.002 & 0.006 & 0 & 0.004 & 0.010 \\
\hline
\end{tabular}

Reference pore radius $\left(r_{\mathrm{o}}\right)$ is described by a (truncated) log-normal distribution and the remaining model parameters are described by uniform distributions (Case $c$ ). Values of each metric identifying the most influential parameters are reported in bold

adsorbed molecules $(\kappa)$ gains importance with respect to previous cases, quantitatively impacting the pdf of $J$ to an extent which is similar to what exhibited by temperature. This feature is attributed to the abundance of small pores in this scenario, which favors the dominance of the surface diffusion flow mechanism (linked to parameter $\kappa$ ).

Figure 2 depicts the first four statistical moments of methane flow conditioned on values of influential uncertain parameters for Case $c$ (see Table 4). Unconditional moments are also shown as a reference. Overall, the results are qualitatively similar to those embedded in Fig. 1 for Case $a$. The unconditional mean and variance of $J$ in Case $c$ are reduced (to approximately one-fourth and one-sixth, respectively) with respect to the corresponding values for Case $a$. Otherwise, unconditional skewness and kurtosis increase by about 2.6 and 6 times, respectively. These behaviors are attributed to the larger frequency of small reference pore radius values considered in Case $c$ with respect to Case $a$ (and $b$ ). Low values of reference pore radius are associated with large values of surface diffusion and to small values of mean and variance of methane flow. Conditioning on $r_{\mathrm{o}}$ and $\phi_{\mathrm{o}}$ imprints variations to the model output mean and variance across the entire range of variability of these parameters (Fig. 2). We further note that conditioning on $r_{\mathrm{o}}$ strongly reduces skewness and kurtosis of the pdf of $J$, thus reducing the probability associated with extreme (large) values of $J$.

Conditioning on $p$ induces variations in the (first four) statistical moments of the model output. Conditioning on larger values of this quantity yields the highest values of mean and variance of the model output. A minimum in the values of conditional variance, skewness, and kurtosis is observed in the interval $1 \mathrm{MPa}<p<15 \mathrm{MPa}$. Finally, the blockage/migration ratio of adsorbed molecules displays (a small but noticeable) influence on the model output pdf. Mean and variance of $J$ decrease with increasing values of $\kappa$. This behavior is expected, given the nature of $\kappa$, high values of this parameter being related to significant blockage of gas molecules on the geomaterial surface. 

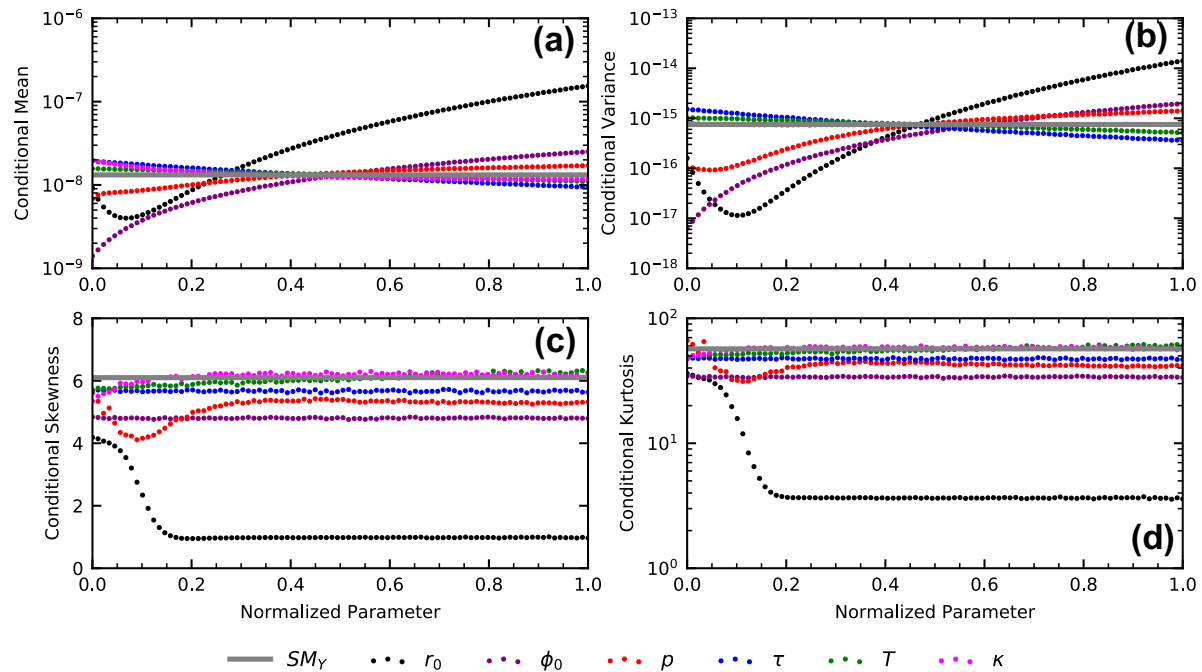

Fig. 2 First four statistical moments of methane flow $J\left(\mathrm{~T} / \mathrm{m}^{2}\right.$ year) conditional on values of the most influential model parameters (see Table 4): a expected value, b variance, c skewness, and d kurtosis. The corresponding unconditional moments (i.e., $\mathrm{SM}_{Y}$ ) are also depicted (gray bold horizontal lines). Intervals of variation of the uncertain model parameters are rescaled within the unit interval for graphical representation purposes. Note that $r_{\mathrm{o}}$ is described by a truncated log-normal pdf and the remaining model parameters are described by uniform pdfs (Case $c$ )

\subsection{Scaling of Gas Flow Model and Identification of Dominant Flow Mechanisms}

A pure diffusion modeling approach has been shown to represent with an acceptable degree of accuracy the movement of methane in low-permeability media ( $\mathrm{Lu}$ et al. 2015). Such a model embeds all physics governing the system dynamics in a unique parameter (i.e., a diffusion coefficient $D$ ) and, under steady-state conditions, the mass flow rate of methane can be expressed as

$$
J_{d}=-D \frac{\partial C}{\partial l},
$$

where $\partial C / \partial l$ represents the spatial gradient of methane concentration $(C)$, i.e., the driving force of the system. Considering an isothermal system under single-phase flow and introducing the density of methane, $\rho=p M / R T Z$, Eq. (11) can be written as

$$
J_{d}=-\frac{D M}{R T Z}\left(1-\frac{p}{Z} \frac{\mathrm{d} Z}{\mathrm{~d} p}\right) \frac{\partial p}{\partial l} .
$$

We complete our set of results and discussion by noting that the model illustrated in Sect. 2.1 coincides with a pure diffusion model (Eq. (12)) under single-phase conditions, as we illustrate in the following.

Equation (1) can be written as

$$
J=-B \frac{\partial p}{\partial l},
$$

with $B=B_{\mathrm{v}}+B_{\mathrm{k}}+B_{\mathrm{ss}}$, where 


$$
\begin{aligned}
& B_{\mathrm{v}}=w_{\mathrm{v}} \zeta_{\mathrm{mb}} \frac{r^{2} p M}{8 \eta Z R T}\left(1+\alpha K_{\mathrm{n}}\right)\left(1+\frac{4 K_{\mathrm{n}}}{1+K_{\mathrm{n}}}\right), \\
& B_{\mathrm{k}}=w_{\mathrm{k}} \frac{2}{3} \zeta_{\mathrm{mb}} r \delta^{D_{\mathrm{f}}-2}\left(\frac{8 Z M}{\pi R T}\right)^{1 / 2} \frac{p}{Z} C_{\mathrm{g}} \\
& B_{\mathrm{ss}}=\zeta_{\mathrm{ms}} \frac{D_{\mathrm{s}} C_{\mathrm{sc}}}{p}
\end{aligned}
$$

Comparing Eqs. (12) and (13), it can be seen that the diffusion coefficient $D$ can be decomposed according to each flow mechanism as

$$
D=D_{\mathrm{v}}+D_{\mathrm{k}}+D_{\mathrm{ss}}
$$

with

$$
D_{i}=\frac{B_{i} R T Z}{M\left(1-\frac{p}{Z} \frac{\mathrm{d} Z}{\mathrm{~d} p}\right)},
$$

where $i=\mathrm{v}, \mathrm{k}$, ss. Note that we introduce three effective diffusion coefficients in Eq. (15). These are associated with the slip flow $\left(D_{\mathrm{v}}\right)$, the Knudsen diffusion $\left(D_{\mathrm{k}}\right)$, and the surface diffusion $\left(D_{\mathrm{ss}}\right)$ components of model (1), respectively, and, to the best of our knowledge, are new for the flow model considered in this work. The variety of mechanisms included in model (1) are fully encapsulated in an overall diffusion coefficient $D$ as illustrated in Eqs. (12), (15), and (16), where the contribution of each of the processes described in Sect. 2.1 is clearly recognizable.

Figure 3 depicts color maps quantifying the relative strength of the contribution of the three flow mechanism (slip flow in red, Knudsen diffusion in green, and surface diffusion in blue) to the overall diffusion coefficient defined by Eq. (15) considering various combinations of all uncertain parameters embedded in Eq. (1) for all scenarios investigated. Each sub-plot depicts the average value of the ratio $D_{i} / D$ as a function of two parameters (i.e., averaging is performed with respect to uncertain parameters with the exception of the two varying along the (normalized) axes of the subplots), selected among those which were classified as most influential to the system (see Sects. 3.1 and 3.2).

Our results indicate that the dominant flow mechanism in defining the overall diffusion coefficient (and consequently the methane flow) is slip flow (in red in Fig. 3) in all of the analyzed cases. An exception is observed for small values of the reference pore radius and/ or small pore pressure, where surface diffusion is dominant. The contribution of Knudsen diffusion mechanism is always negligible. This suggests that it is possible to simplify Eq. (1) by neglecting the Knudsen diffusion mechanism in the evaluation of methane flow.

Further simplifications of the methane flux model illustrated in Sect. 2.1 can be considered when the dominance of a given flow mechanism can be clearly established. For example, Fig. 3 suggests that the identification of the dominant flow mechanism is affected by the pdf of the uncertain model parameters. If $r_{\mathrm{o}}$ is represented by a Gaussian (or uniform) pdf, $J$ is mainly dominated by slip flow or surface diffusion with a sharp transition zone between these two mechanisms. Otherwise, when $r_{\mathrm{o}}$ is represented by a log-normal pdf both mechanisms (i.e., slip flow and surface diffusion) may play an important role in the estimation of methane migration independent of the value of the model parameters.

Finally, we evaluate the pdf of the overall diffusion coefficient $(D)$ by making use of Eqs. (15) and (16) for all scenarios analyzed. Sample pdfs as well as corresponding 

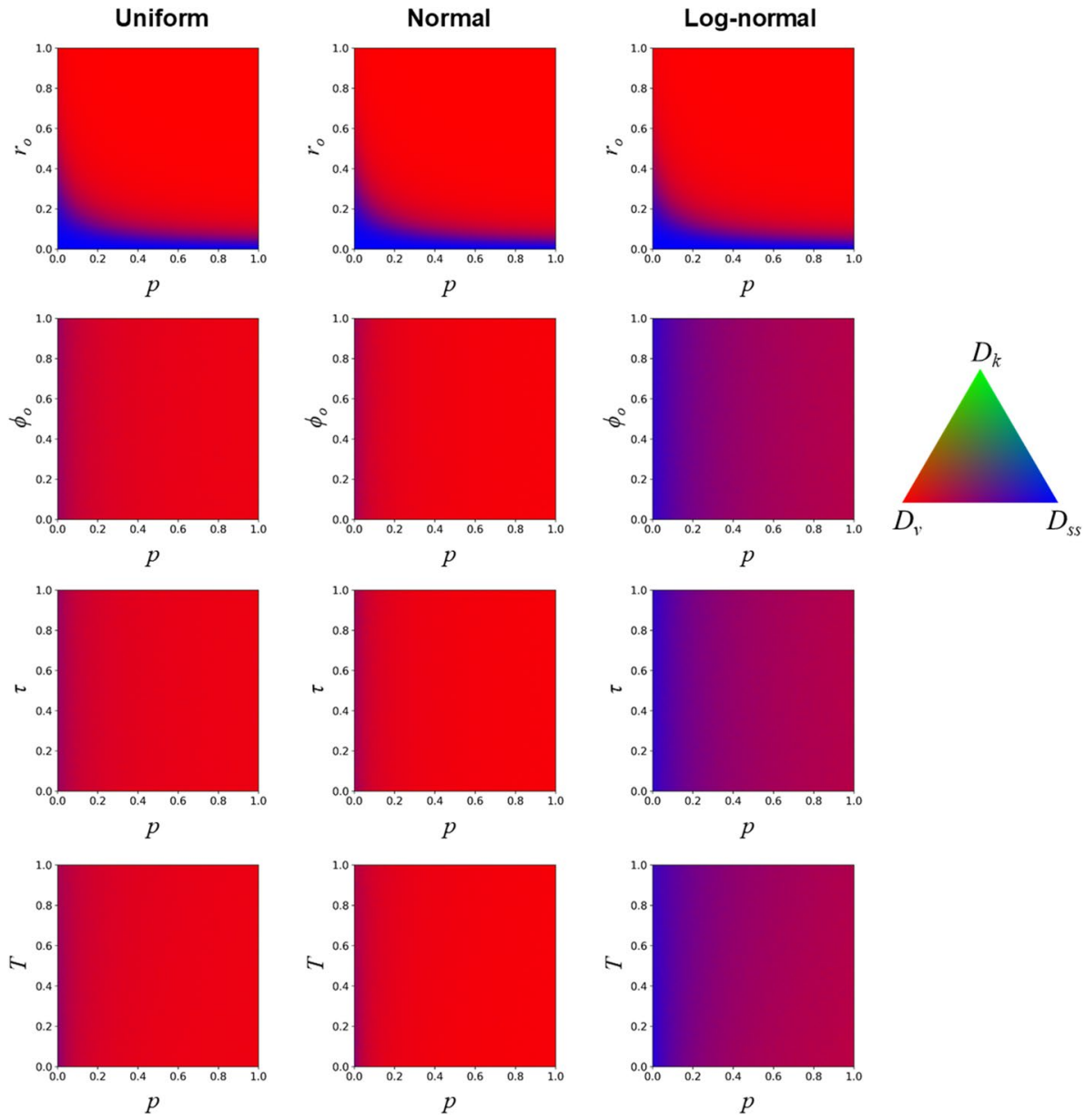

Fig. 3 Relative contribution of the effective diffusion coefficients $\left(D_{\mathrm{v}}, D_{\mathrm{k}}\right.$, and $\left.D_{\mathrm{ss}}\right)$ to the overall diffusion coefficient $D$ rendered by Eq. (15) and (16). Intervals of variation of the uncertain model parameters are rescaled within the unit interval for graphical representation purposes

Maximum Likelihood (ML) fits of log-normal distributions are depicted in Fig. 4 in logarithmic and natural scales. Positive skewness and large kurtosis are evident for all cases, these being larger for Case $c$, as illustrated in Sect. 3.2. These results reinforce the observation of higher frequencies of low $J$ values in Case $c$ with respect to the other settings investigated. Sample statistical moments (mean, variance, coefficient of variation, skewness, and kurtosis) of the pdf of $D$ are listed in Table 5 together with the parameters of the ML-based log-normal models. The overall diffusion coefficient can vary across about four orders of magnitude (i.e., between $10^{-9}$ and $10^{-5} \mathrm{~m}^{2} / \mathrm{s}$ ). As expected, the largest variance of $D$ is associated with Case $a$, where all parameters of model (1) are characterized by uniform pdfs. Otherwise, the largest coefficient of variation of $D$ is associated with Case $c$. Finally, we remark that the results embedded in Fig. 4 can be of practical assistance, as they allow for fast evaluations of the probability that methane flow in low-permeability media exceeds a given threshold value. 

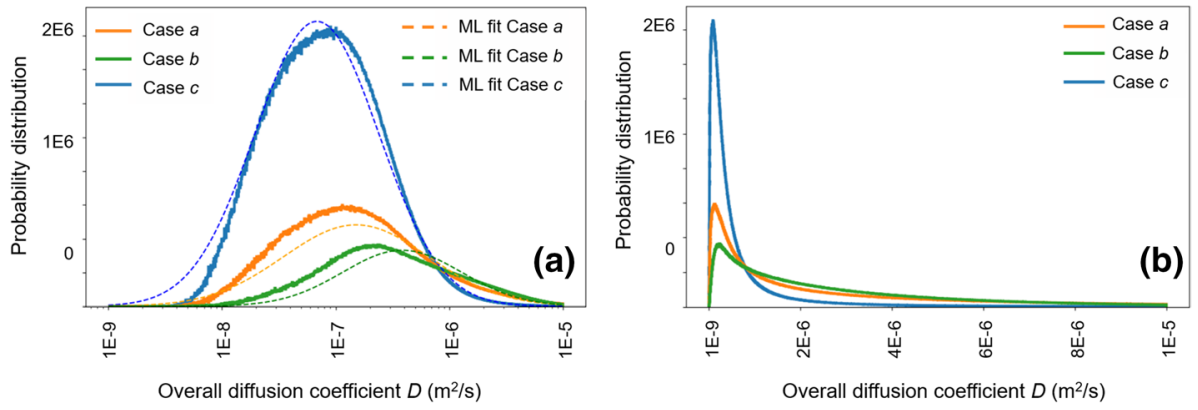

Fig. 4 Probability density functions (in logarithmic (a) and natural (b) scale) of the overall diffusion coefficient rendered by Eq. (15) for model parameters characterized by (i) uniform distributions (Case $a$ ), (ii) truncated normal distributions (Case $b$ ), and (iii) uniform distributions with the exception of $r_{\mathrm{o}}$ which is represented by a log-normal distribution (Case $c$ ). Dashed curves represent a ML-based fit with a log-normal model for each case

Table 5 Sample mean, variance, coefficient of variation, skewness, and kurtosis of the overall diffusion coefficient $D$ $\left(\mathrm{m}^{2} / \mathrm{s}\right)($ Eq. $(15))$ together with parameters of log-normal models ( $\mu$ and $\sigma$ ) evaluated through ML fits against sample pdfs

\begin{tabular}{llll}
\hline Feature & Case $a$ & Case $b$ & Case $c$ \\
\hline Mean $\left(\times 10^{-6}\right)$ & 3.14 & 2.96 & 0.78 \\
Variance $\left(\times 10^{-12}\right)$ & 16.3 & 9.53 & 2.46 \\
CV & 1.29 & 1.04 & 2.01 \\
Skewness & 2.21 & 1.95 & 5.90 \\
Kurtosis & 9 & 8.1 & 53.54 \\
$\mu$ & -13.53 & -13.31 & -14.92 \\
$\sigma$ & 1.47 & 1.21 & 1.26 \\
\hline
\end{tabular}

\section{Conclusions}

We perform a rigorous Global Sensitivity Analysis (GSA) to assess the impact of uncertain model parameters on the evaluation of methane flow $(J)$ in low-permeability media, such as caprocks. We study three scenarios that consider differing characterizations of the probability density function (pdf) describing model uncertain parameters to assess the impact of this choice on the results of the analysis. In details, we consider settings according to which (i) all model parameters are represented through uniform pdfs, (ii) all model parameters are represented through (truncated) Gaussian pdfs, and (iii) the reference pore radius is characterized by a (truncated) log-normal pdf, all remaining parameters being associated with uniform pdfs.

Our work leads to the following main conclusions:

1. The uncertainty of methane flow is governed by uncertainty in the reference pore radius, followed (in decreasing order of importance) by reference porosity, pore pressure, tortuosity, temperature, and (to a lesser extent) blockage migration ratio of adsorbed molecules. The remaining parameters of the investigated model (Sect. 2.1) being practically uninfluential. This result can assist future efforts to allocate resources during experimental activities aimed at characterizing methane flow in caprocks. 
2. The gas flow model introduced by Wu et al. (2016) (Sect. 2.1) can be related to a simple pure diffusion model by introducing an overall diffusion coefficient $(D)$. The latter represented by the contribution of three effective diffusion coefficients, each associated with a well-defined flow mechanism. The ensuing mathematical structure of $D$ allows distinguishing the relative contribution of all flow mechanisms to the overall methane flow. The relationship we derive also enables one to estimate the pdf of $D$ when the model parameters are uncertain. The latter is a useful tool which can assist the probabilistic evaluation of $J$ even in the absence of the detailed amount of information which is typically required to characterize the full methane flow model.

3. The shape of the pdf employed to characterize uncertain model parameters affects the results of our GSA. Additionally, it has a marked effect in the definition of the dominating transport mechanisms of the model. With reference to the model parameter variability considered in this study, as evaluated on the basis of available information, our results suggest that the dominant transport mechanism is slip flow. Surface diffusion plays also an important role, especially for low values of reference pore radius and pore pressure, while Knudsen diffusion is negligible in all of the test cases analyzed.

\section{Appendix: Additional mathematical details related to the description of the gas flow model introduced in Sect. 2.1}

The correction factor $\zeta_{\mathrm{ms}}$ is given by

$$
\zeta_{\mathrm{ms}}=\frac{\phi}{\tau}\left(1-\frac{r_{\mathrm{ad}}}{r}\right)^{2}\left[\left(1-\frac{r_{\mathrm{ad}}}{r}\right)^{-2}-1\right],
$$

with

$$
\begin{gathered}
r_{\mathrm{ad}}=r-d_{\mathrm{m}} \theta, \\
r=r_{\mathrm{o}}\left(\frac{p_{c}-p}{p_{\mathrm{o}}}\right)^{-t}, \\
\phi=\phi_{\mathrm{o}}\left(\frac{p_{c}-p}{p_{\mathrm{o}}}\right)^{-q},
\end{gathered}
$$

where $r_{\mathrm{ad}}$ is thickness of the adsorbed gas layer, $d_{\mathrm{m}}$ is gas molecule diameter, $p_{c}$ is overburden pressure, $p_{\mathrm{o}}$ is atmospheric pressure, and $\theta$ is evaluated through a Langmuir equilibrium isotherm as

$$
\theta=\frac{p / Z}{p_{\mathrm{L}}+p / Z},
$$

where $p_{\mathrm{L}}$ is a Langmuir pressure evaluated with

$$
p_{\mathrm{L}}=p_{L_{\mathrm{o}}} \exp \left(-\frac{\Delta H}{R T}\right) .
$$

The correction factor $\zeta_{\mathrm{mb}}$ is expressed as 


$$
\zeta_{\mathrm{mb}}=\frac{\phi}{\tau}\left(1-\frac{r_{\mathrm{ad}}}{r}\right)^{2}
$$

The value of $\alpha$ (in Eq. (7)) is evaluated through

$$
\alpha=\alpha_{0} \frac{2}{\pi} \tan ^{-1}\left(\alpha_{1} K_{n}^{\beta}\right),
$$

where uncertain parameters $\alpha_{0}, \alpha_{1}$, and $\beta$ allow representing the variation of $\alpha$ as a function of the Knudsen number $\left(K_{\mathrm{n}}\right)$. Here, $\alpha_{0}$ represents the maximum value of $\alpha$ for large values of $K_{\mathrm{n}}, \alpha_{1}$ governs the values of $\alpha$ for small values of $K_{\mathrm{n}}$, and $\beta$ is a shape parameter.

The surface diffusion coefficient $\left(D_{\mathrm{s}}\right)$ is given by

$$
D_{\mathrm{s}}=D_{\mathrm{s}}^{0} \frac{(1-\theta)+\frac{\kappa}{2} \theta(2-\theta)+H(1-\kappa)(1-\kappa) \frac{\kappa}{2} \theta^{2}}{\left(1-\theta+\frac{\kappa}{2} \theta\right)^{2}},
$$

with

$$
\begin{gathered}
D_{\mathrm{s}}^{0}=8.29 \times 10^{-7} \exp \left(-\frac{\Delta H^{0.8}}{R T}\right), \\
H(1-\kappa)=\left\{\begin{array}{l}
0, \text { if } \kappa \geq 1 \\
1,0 \leq \kappa \leq 1
\end{array} .\right.
\end{gathered}
$$

The adsorbed concentration $\left(C_{\mathrm{sc}}\right)$ is given by

$$
C_{\mathrm{sc}}=\frac{4 \theta M}{\pi d_{\mathrm{m}}^{3} N_{A}}
$$

where $N_{A}$ is the Avogadro Constant $\left(6.02 \times 10^{-23} / \mathrm{mol}\right)$.

Funding Open access funding provided by Politecnico di Milano within the CRUI-CARE Agreement. The authors acknowledge financial support from Geolog srl.

Data Availability All data used in the paper will be retained by the authors for at least 5 years after publication and will be available to the readers upon request.

Code Availability Codes used in this paper are available in the following github repository https://github. com/rlsandovalp/Sensitivity_Analysis.

\section{Declarations}

\section{Conflict of interest Not applicable}

Open Access This article is licensed under a Creative Commons Attribution 4.0 International License, which permits use, sharing, adaptation, distribution and reproduction in any medium or format, as long as you give appropriate credit to the original author(s) and the source, provide a link to the Creative Commons licence, and indicate if changes were made. The images or other third party material in this article are included in the article's Creative Commons licence, unless indicated otherwise in a credit line to the material. If material is not included in the article's Creative Commons licence and your intended use is not permitted by statutory regulation or exceeds the permitted use, you will need to obtain permission directly from the copyright holder. To view a copy of this licence, visit http://creativecommons.org/licenses/by/4.0/. 


\section{References}

Bianchi Janetti, E., Guadagnini, L., Riva, M., Guadagnini, A.: Global sensitivity analyses of multiple conceptual models with uncertain parameters driving groundwater flow in a regional-scale sedimentary aquifer. J. Hydrol. 574, 544-556 (2018). https://doi.org/10.1016/j.jhydrol.2019.04.035

Ceresa, L., Guadagnini, A., Porta, G.M., Riva, M.: Formulation and probabilistic assessment of reversible biodegradation pathway of Diclofenac in groundwater. Water Res. 204(117), 466 (2021). https://doi. org/10.1016/J.WATRES.2021.117466

Chiquet, P., Daridon, J.L., Broseta, D., Thibeau, S.: CO2/water interfacial tensions under pressure and temperature conditions of CO2 geological storage. Energy Convers. Manag. 48(3), 736-744 (2007). https://doi.org/10.1016/J.ENCONMAN.2006.09.011

Ciriello, V., Guadagnini, A., Di Federico, V., Edery, Y., Berkowitz, B.: Comparative analysis of formulations for conservative transport in porous media through sensitivity-based parameter calibration. Water Resour. Res. 49(9), 5206-5220 (2013). https://doi.org/10.1002/wrcr.20395

Civan, F.: Effective correlation of apparent gas permeability in tight porous media. Transp. Porous Media 82(2), 375-384 (2010). https://doi.org/10.1007/s11242-009-9432-z

Colombo, I., Porta, G.M., Ruffo, P., Guadagnini, A.: Uncertainty quantification of overpressure buildup through inverse modeling of compaction processes in sedimentary basins. Hydrogeol. J. 25(2), 385403 (2017). https://doi.org/10.1007/s10040-016-1493-9

Coppens, M.O.: The effect of fractal surface roughness on diffusion and reaction in porous catalysts-from fundamentals to practical applications. Catal. Today 53(2), 225-243 (1999). https://doi.org/10.1016/ S0920-5861(99)00118-2

Coppens, M.O., Dammers, A.J.: Effects of heterogeneity on diffusion in nanopores-from inorganic materials to protein crystals and ion channels. Fluid Phase Equilib. 241(1-2), 308-316 (2006). https://doi.org/10. 1016/J.FLUID.2005.12.039

Darabi, H., Ettehad, A., Javadpour, F., Sepehrnoori, K.: Gas flow in ultra-tight shale strata. J. Fluid Mech. 710, 641-658 (2012). https://doi.org/10.1017/jfm.2012.424

Dell'Oca, A., Riva, M., Guadagnini, A.: Moment-based metrics for global sensitivity analysis of hydrological systems. Hydrol. Earth Syst. Sci. 21(12), 6219-6234 (2017). https://doi.org/10.5194/ hess-21-6219-2017

Dell'Oca, A., Riva, M., Guadagnini, A.: Global sensitivity analysis for multiple interpretive models with uncertain parameters. Water Resour. Res. 56(2), 1-20 (2020). https://doi.org/10.1029/2019WR025754

Dembicki-Jr, H.: Petroleum geochemistry for exploration and production. Candice Janco (2017). https://doi. org/10.1016/j.jhsb.2006.10.005

Dong, J.J., Hsu, J.Y., Wu, W.J., Shimamoto, T., Hung, J.H., Yeh, E.C., Wu, Y.H., Sone, H.: Stress-dependence of the permeability and porosity of sandstone and shale from TCDP Hole-A. Int. J. Rock Mech. Min. Sci. 47(7), 1141-1157 (2010). https://doi.org/10.1016/J.IJRMMS.2010.06.019

Hughes, J.D.: Energy: a reality check on the shale revolution. Nature 494(7437), 307-308 (2013). https:// doi.org/10.1038/494307a

Javadpour, F., Singh, H., Rabbani, A., Babaei, M., Enayati, S.: Gas flow models of shale: a review. Energy Fuels 35(4), 2999-3010 (2021). https://doi.org/10.1021/acs.energyfuels.0c04381

Karniadakis, G., Beskok, A., Aluru, N.: Microflows and Nanoflows. Springer, Berlin (2005)

Koks, E.E., Bočkarjova, M., de Moel, H., Aerts, J.C.: Integrated direct and indirect flood risk modeling: development and sensitivity analysis. Risk Anal. 35(5), 882-900 (2015). https://doi.org/10.1111/risa. 12300

la Cecilia, D., Porta, G.M., Tang, F.H., Riva, M., Maggi, F.: Probabilistic indicators for soil and groundwater contamination risk assessment. Ecol. Ind. 115(106), 424 (2020). https://doi.org/10.1016/j.ecolind. 2020.106424

Laloy, E., Rogiers, B., Vrugt, J.A., Mallants, D., Jacques, D.: Efficient posterior exploration of a highdimensional groundwater model from two-stage Markov chain Monte Carlo simulation and polynomial chaos expansion. Water Resour. Res. 49(5), 2664-2682 (2013). https://doi.org/10.1002/wrcr.20226

Lemmon, E.W., Bell, I., Huber, M.L., McLinden, M.O.: NIST Standard Reference Database 23: Reference Fluid Thermodynamic and Transport Properties-REFPROP, Version 10.0, National Institute of Standards and Technology (2018).https://doi.org/10.18434/T4/1502528

Li, Z., Dong, M., Li, S., Huang, S.: CO2 sequestration in depleted oil and gas reservoirs-caprock characterization and storage capacity. Energy Convers. Manage. 47(11-12), 1372-1382 (2006). https://doi.org/ 10.1016/J.ENCONMAN.2005.08.023

Liu, J., Wang, J.G., Gao, F., Ju, Y., Zhang, X., Zhang, L.C.: Flow consistency between non-darcy flow in fracture network and nonlinear diffusion in matrix to gas production rate in fractured shale gas reservoirs. Transp. Porous Media 111(1), 97-121 (2016). https://doi.org/10.1007/s11242-015-0583-9 
Lu, J., Larson, T.E., Smyth, R.C.: Carbon isotope effects of methane transport through Anahuac Shale: a core gas study. J. Geochem. Explor. 148, 138-149 (2015). https://doi.org/10.1016/j.gexplo.2014. 09.005

Maina, F.Z., Siirila-Woodburn, E.R.: The role of subsurface flow on evapotranspiration: a global sensitivity analysis. Water Resour. Res. 56(7), 1-20 (2020). https://doi.org/10.1029/2019WR026612

Maina, F.Z., Guadagnini, A., Riva, M.: Impact of multiple uncertainties on gravimetric variations across randomly heterogeneous aquifers during pumping. Adv. Water Resour. 154(103), 978 (2021). https://doi.org/10.1016/J.ADVWATRES.2021.103978

Mehmani, A., Prodanović, M., Javadpour, F.: Multiscale, multiphysics network modeling of shale matrix gas flows. Transp. Porous Media 99(2), 377-390 (2013). https://doi.org/10.1007/ s11242-013-0191-5

Mohd Amin, S., Weiss, D.J., Blunt, M.J.: Reactive transport modelling of geologic CO2 sequestration in saline aquifers: the influence of pure $\mathrm{CO} 2$ and of mixtures of $\mathrm{CO} 2$ with $\mathrm{CH} 4$ on the sealing capacity of cap rock at $37^{\circ} \mathrm{C}$ and 100bar. Chem. Geol. 367, 39-50 (2014). https://doi.org/10.1016/J.CHEMG EO.2014.01.002

Naraghi, M.E., Javadpour, F., Ko, L.T.: An object-based shale permeability model: non-Darcy gas flow, sorption, and surface diffusion effects. Transp. Porous Media 125(1), 23-39 (2018). https://doi.org/ 10.1007/s11242-017-0992-Z

Pan, Z., Connell, L.D., Camilleri, M., Connelly, L.: Effects of matrix moisture on gas diffusion and flow in coal. Fuel 89(11), 3207-3217 (2010). https://doi.org/10.1016/j.fuel.2010.05.038

Rani, S., Prusty, B.K., Pal, S.K.: Adsorption kinetics and diffusion modeling of CH4 and CO2 in Indian shales. Fuel 216, 61-70 (2017). https://doi.org/10.1016/j.fuel.2017.11.124

Saltelli, A., Sobol', I.M.: Sensitivity analysis for nonlinear mathematical models: numerical experience (in Russian). Math. Models Comput. Exp. 7(11), 16-28 (1995)

Saltelli, A., Annoni, P., Azzini, I., Campolongo, F., Ratto, M., Tarantola, S.: Variance based sensitivity analysis of model output. Design and estimator for the total sensitivity index. Comput. Phys. Commun. 181(2), 259-270 (2010)

Schloemer, S., Krooss, B.M.: Molecular transport of methane, ethane and nitrogen and the influence of diffusion on the chemical and isotopic composition of natural gas accumulations. Geofluids 4(1), 81-108 (2004). https://doi.org/10.1111/j.1468-8123.2004.00076.x

Schlömer, S., Krooss, B.M.: Experimental characterisation of the hydrocarbon sealing efficiency of cap rocks. Mar. Pet. Geol. 14(5), 565-580 (1997). https://doi.org/10.1016/S0264-8172(97)00022-6

Setzmann, U., Wagner, W.: A new equation of state and tables of thermodynamic properties for methane covering the range from the melting line to $625 \mathrm{~K}$ at pressures up to $100 \mathrm{MPa}$. J. Phys. Chem. Ref. Data 20(6), 1061-1155 (1991). https://doi.org/10.1063/1.555898

Singh, H., Myong, R.S.: Critical review of fluid flow physics at micro- to nano-scale porous media applications in the energy sector. Adv. Mater. Sci. Eng. (2018). https://doi.org/10.1155/2018/9565240

Sochala, P., Le Maître, O.P.: Polynomial Chaos expansion for subsurface flows with uncertain soil parameters. Adv. Water Resour. 62, 139-154 (2013). https://doi.org/10.1016/j.advwatres.2013.10.003

Song, W., Yao, J., Li, Y., Sun, H., Zhang, L., Yang, Y., Zhao, J., Sui, H.: Apparent gas permeability in an organic-rich shale reservoir. Fuel 181, 973-984 (2016). https://doi.org/10.1016/j.fuel.2016.05.011

Sun, Z., Li, X., Shi, J., Zhang, T., Sun, F.: Apparent permeability model for real gas transport through shale gas reservoirs considering water distribution characteristic. Int. J. Heat Mass Transf. 115, 1008-1019 (2017). https://doi.org/10.1016/j.ijheatmasstransfer.2017.07.123

Tan, Y., Pan, Z., Liu, J., Kang, J., Zhou, F., Connell, L.D., Yang, Y.: Experimental study of impact of anisotropy and heterogeneity on gas flow in coal. Part I: diffusion and adsorption. Fuel 232(15), 444-453 (2018). https://doi.org/10.1016/j.fuel.2018.05.173

US Energy Information Administration (2015) World Shale Resource Assessments. https://www.eia.gov/ analysis/studies/worldshalegas/

Wang, T., Tian, S., Li, G., Zhang, P.: Analytical model for real gas transport in shale reservoirs with surface diffusion of adsorbed gas. Ind. Eng. Chem. Res. 58(51), 481-489 (2019). https://doi.org/10. 1021/acs.iecr.9b05630

Wu, K., Chen, Z., Li, X.: Real gas transport through nanopores of varying cross-section type and shape in shale gas reservoirs. Chem. Eng. J. 281, 813-825 (2015a). https://doi.org/10.1016/j.cej.2015.07.012

Wu, K., Li, X., Wang, C., Yu, W., Chen, Z.: Model for surface diffusion of adsorbed gas in nanopores of shale gas reservoirs. Ind. Eng. Chem. Res. 54(12), 3225-3236 (2015b). https://doi.org/10.1021/ ie $504030 \mathrm{v}$

Wu, K., Chen, Z., Li, X., Guo, C., Wei, M.: A model for multiple transport mechanisms through nanopores of shale gas reservoirs with real gas effect-adsorption-mechanic coupling. Int. J. Heat Mass Transf. 93, 408-426 (2016). https://doi.org/10.1016/j.ijheatmasstransfer.2015.10.003 
Wu, K., Chen, Z., Li, X., Xu, J., Li, J., Wang, K., Wang, H., Wang, S., Dong, X.: Flow behavior of gas confined in nanoporous shale at high pressure: real gas effect. Fuel 205, 173-183 (2017). https://doi.org/ 10.1016/j.fuel.2017.05.055

Xiao, S., Praditia, T., Oladyshkin, S., Nowak, W.: Global sensitivity analysis of a $\mathrm{CaO} / \mathrm{Ca}(\mathrm{OH}) 2$ thermochemical energy storage model for parametric effect analysis. Appl. Energy 285, 116 (2021). https:// doi.org/10.1016/j.apenergy.2021.116456

Yuan, W., Pan, Z., Li, X., Yang, Y., Zhao, C., Connell, L.D., Li, S., He, J.: Experimental study and modelling of methane adsorption and diffusion in shale. Fuel 117, 509-519 (2014). https://doi.org/10.1016/j. fuel.2013.09.046

Zhang, Q., Su, Y., Wang, W., Lu, M., Sheng, G.: Gas transport behaviors in shale nanopores based on multiple mechanisms and macroscale modeling. Int. J. Heat Mass Transf. 125, 845-857 (2018). https://doi. org/10.1016/j.ijheatmasstransfer.2018.04.129

Ziarani, A.S., Aguilera, R.: Knudsen's permeability correction for tight porous media. Transp. Porous Media 91(1), 239-260 (2012). https://doi.org/10.1007/s11242-011-9842-6

Publisher's Note Springer Nature remains neutral with regard to jurisdictional claims in published maps and institutional affiliations. 